\title{
DESIDERATA FOR FK5
}

\author{
W. Fricke and W. GLIESE
}

\section{Purpose of a Fundamental Catalogue}

A fundamental catalogue compiled from independent and differential observations of stellar positions at various epochs provides the fundamental system of positions and proper motions of the stars. The system ought to be compiled in such a way that it represents the inertial frame of reference as accurately as practicable. Its direct purpose is to serve as the reference system of positional astronomy. It should fulfil the requirements of astronomical research, in particular in celestial mechanics and galactic research, as well as the demands of the astronomical determinations of time and of latitudes. It has also to fulfil the requirements of geodesy and navigation. For all these purposes the system must be uniform over the whole sky (free from regional systematic errors), and it has to be uniform over a large range of stellar magnitudes (free from errors depending on the magnitude of the stars).

\section{Desired Stellar Composition of a New Fundamental Catalogue}

The FK4 contains 1535 stars brighter than magnitude 7.5. The limitation to bright stars was essentially caused by the optical limit of older meridian circles. The fundamental stars were chosen on the basis of the number of accurate independent observations available per star, and they were selected such that an approximately uniform distribution of fundamental stars exists over the sky.

In further improvements of the fundamental catalogue the number of available independent observations at different instruments will remain one of the important criteria for the selection of more stars. The principle of uniform distribution may, however, no longer be used to exclude stars with observations of high quality. Furthermore, the number of fundamental stars may not be limited owing to the amount of work involved in the compilation of a fundamental catalogue. The data processing may be done by means of electronic computers.

Observations available at the present time suggest, first, a considerable increase of the number of fundamental stars and, second, the extension of the system down to the magnitude limit of about $9 \cdot 0$. Only with the completion of the processing of the observational data will one be able to decide which stars can be included in the fundamental catalogue from the view of systematic accuracy. It is fairly certain that the total number of suitable stars in the whole sky will scarcely exceed about 5000 objects.

Perek (ed.), Highlights of Astronomy, 301-305. (c) I.A.U. 
There will remain many stars observed by differential techniques at three or more epochs with respect to some system which can be reduced satisfactorily to the fundamental system. These stars, however, will not attain the accuracy of the fundamental stars. The number of these stars may be of the order of 30000 , and their positions and proper motions may form a new general catalogue. In a new general catalogue the requirements of galactic research have to be carefully taken into account. The principle of a uniform distribution of the catalogue stars over the sky can no longer be followed, since it would not be wise to exclude stars with accurate data in the galactic belt. More attention has also to be given to objects of special interest in galactic research. Valuable impetus was given in this direction by such lists as that compiled by Blaauw.

For both catalogues, the fundamental and the general, the determination of the system free from magnitude errors down to magnitude $9 \cdot 0$ is the main problem.

\section{Recent Observations Relevant to the Improvement of the FK4 System}

\section{A. LIST OF NEW OBSERVATIONS}

The communication of observations which observers regard as relevant to the improvement of the Fourth Fundamental Catalogue is of great importance. A list of publications is presented here containing recent observations which will have to be taken into account in improving the FK 4 system. (The list does not contain observations made by differential techniques.) Should the list be incomplete, we should be glad to receive more information.

(a) Astrolabe observations:

Neuchâtel: Publ. Obs. Neuchâtel, 9, 1959.

Paris: $\quad$ Bull. Astron., 23, 1961, 343.

Curaçao: $\quad$ Netherlands Geod. Comm., 1, 1963, No. 4.

Zi-Ka-Wei: $\quad$ Acta Astr. Sinica, 11, 1963, 73.

Potsdam: $\quad$ Arbeiten Geod. Inst. Potsdam, 12, 1966.

Tananarive: J.d. Obs., 49, 1966, 387.

Herstmonceux: $\}$

Cape: $\}$ private comm., D.V. Thomas, 1967.

Some of these papers contain data of little more than one hundred FK4 stars; in these cases the systematic relation between the observations and the FK4 cannot be determined very reliably.

(b) Observations of the Time Service in U.S.S.R.:

Catalogue $\phi 2: \quad$ Astr. Zu., 34, 1957, 613.

$\phi 6^{\prime}:$ Izv. Glav. Astr. Obs. Pulkovo, 22, 1961, 2.

ф678: Trud. Glav. Astr. Obs. Pulkovo (II), 75, 1966, 29. 
(c) Catalogues observed with classical meridian instruments:

$\mathrm{W}_{50}$ : Publ. U.S.N.O., 19, 1964, 1.

$\mathrm{Pu}_{60}$ : Izv. Glav. Astr. Obs. Pulkovo, No. 179, 1966, 3.

$\mathrm{Pu}_{50} \mathrm{~F}$ : Trud. Glav. Astr. Obs. Pulkovo (II), 76, 1966, 5.

(d) Dynamical coordinate system derived from minor planet observations: Ann. Tokyo Obs. (II), 10, 1967, 113.

(e) Right ascension system of the southern polar cap:

Cerro Calan: Astr. Zu., 42, 1965, 823.

Cape Cat. of Circumpolar Stars: Roy. Obs. Bull., 106, 1966.

\section{B. ERRORS OF THE FK4 SYSTEMS INDICATED BY RECENT OBSERVATIONS}

(a) Right ascension system:

$\Delta \alpha_{\delta}$ : The mean system of the more recent observations indicates that the FK4 right ascensions between $+5^{\circ}$ and $+50^{\circ}$ require a negative correction which is -0.007 at $+25^{\circ}$.

In the Southern circumpolar region $\left(-70^{\circ}\right.$ to $\left.-85^{\circ}\right)$ both the rigid absolute Cape observations of 1936 and Zverev's observations at Cerro Calan show a deviation from FK4 of about $-0.02 \sec \delta$. According to provisional Cape astrolabe results the fundamental $\alpha_{\delta}$-system south of $-40^{\circ}$ requires a negative correction which becomes more pronounced with decreasing declination reaching $-0.011 \sec \delta$ at $-60^{\circ}$. The sign agrees with that of the Southern observations just mentioned.

$\Delta \alpha_{\alpha}$ : In the zone $+50^{\circ}$ to $+80^{\circ}$ between $11^{\mathrm{h}}$ and $19^{\mathrm{h}}$ the $\alpha_{\alpha}$-system of FK4 obviously requires a positive correction, the maximum being about $+0.008 \sec \delta$ at $16^{\mathrm{h}}$.

(b) Declination system:

Recent observations do not yet permit of any conclusion on deficiencies of the declination system of FK4. The dispersion among the observations is considerable.

\section{UNCONFIRMED ERRORS OF THE FK 4 SYSTEM}

In the equatorial zone the dynamical right ascension system derived by Yasuda from minor-planet observations deviates from FK4 between $+0^{s} .010\left(12^{\mathrm{h}}\right)$ and $-0.015\left(20 / 21^{\mathrm{h}}\right)$. The suggested $\Delta \alpha_{\alpha}$-curve is not confirmed by the catalogues $\mathrm{Pu}_{60}$, $\phi 678$, and $\mathrm{W}_{50}$.

In the whole region from $-30^{\circ}$ to $+75^{\circ}$ the $\delta_{\alpha}$-system of $\mathrm{W}_{50}$ shows a wave against FK4, its maximum being $+0^{\prime \prime} .08$ at $8^{\mathrm{h}}$, its minimum $-0^{\prime \prime} 09$ at $16^{\mathrm{h}}$. While $\mathrm{Pu}_{50} \mathrm{~F}$ and Herstmonceux astrolabe observations appear to confirm these differences, the averaged results from the astrolabe observations at Neuchâtel, Paris, and Potsdam show no remarkable deviations from the FK4 system.

Magnitude equation: The right ascensions of the brightest stars $(\mathrm{m}<2 \cdot 7)$ in $\mathrm{W} 3_{50}$ differ from FK4 by about $-0^{5} .002$ while the photoelectrically observed right ascen- 
sions in $\phi 678$ differ from those in FK4 systematically; the differences increase continuously from -0.006 for stars of magnitude one to +0.003 for stars of magnitude 6 .

D.V. Thomas calls attention to an apparent systematic error in the FK 4 declinations of bright blue stars $(\mathrm{m}<4 \cdot 0): \Delta \delta_{\mathrm{m}, \mathrm{c}}($ Herstm. $-\mathrm{FK} 4)=-0^{\prime \prime} 09$, derived from 12 stars.

\section{Suggestions for Further Observations and the Form of their Presentation}

Suggestions for further observations which may contribute to an improvement of the present fundamental system arise mainly from known deficiencies and suspected shortcomings of the FK4. There are the following main deficiencies:

(1) The foundation of the system South of $-30^{\circ}$ on observations with the Cape meridian circle alone (other instruments in the Southern hemisphere play a minor role in FK4);

(2) the inferior accuracy of the right ascension system compared with that of the declinations; this holds for the whole sky;

(3) regional inhomogeneities in the system over the sky;

(4) the limitation to stars brighter than visual magnitude $7 \cdot 5$.

From these deficiencies we conclude that high priority must be given to the continuation of fundamental observations which are suited to eliminate zonal and regional inhomogeneities. Fundamental observations at more than one instrument in the Southern sky are of eminent importance. For the determination of the zero point in right ascension and for the equator point, observations of the Sun and of major and minor planets are necessary. More attention has to be given to absolute azimuth determinations with meridian circles in order to improve the right ascension system.

Concerning the choice of stars for a fundamental program, we suggest that, in programs covering the sky from one of the poles to about $30^{\circ}$ South or North of the equator, not less than $750 \mathrm{FK} 4$ stars should be included. The desirability of such a large number arises from still existing regional systematic errors. All the other stars in the program should cover the whole magnitude range down to magnitude $9 \cdot 5$, and all necessary care has to be taken for the purpose of eliminating magnitude errors. The inclusion of stars of FK4 Sup, N30 and of Zverev's catalogue of faint fundamental stars is desirable.

So far observations obtained with PZTs and Astrolabes have not contributed to the formation of the fundamental system. In view of the suspected regional systematic errors of the right ascension system in positions and proper motions, the observations with PZTs and Astrolabes may contribute essentially to the compilation of FK5. It is suggested that observers with these instruments present all findings relevant to an improvement of the system in such a way that the observations may be incorporated in FK 5 .

Moreover it is suggested, quite generally, that in the publication of observations the methods of observing - in particular the determination and elimination of in- 
strumental errors - should be clearly described, even if no change in the methods has been made during several decades.

\section{Questions Arising with the Improvement of the FK4}

There are some well-known errors in the proper motions of FK4 preventing the immediate applicability of these data in galactic research. These are the errors caused by incorrect precession and by the unprecessional motion of Newcomb's equinox. At present, these errors are known to an accuracy of about $15 \%$ of their values. If one contemplates a change of the conventional values of precession in the near future, the most favorable opportunity for the change appears to be with the compilation of a new fundamental system. It will be a matter for discussion within the IAU whether the change shall be made with FK5.

Another question concerns the continuation of the traditional technique applied within the FK series of utilizing instrument series alone for the construction of the $\mu_{\alpha}$-system. The continuation of this procedure cannot be recommended. Due account will have to be given to the existence of isolated observations with new instruments. While there is no doubt about the great utility of observations at several epochs with one and the same instrument, 'first epoch' observations with new instruments can play an important part in the improvement of proper motions. In addition, the result of the observations of the time service must be incorporated.

Finally, one may ask, 'When will the time be ripe for the compilation of a new fundamental system?' The answer depends of course on the availability of observations which are not yet incorporated in FK4 and which give sufficient evidence of errors in FK4. At Heidelberg, new methods for comparing catalogues with FK4 have been worked out which make use of electronic computers. Preparations have been made to apply these methods in the computation of the systematic differences between FK4 and important observational catalogues already included in FK4 and between FK4 and new observations. In this way information will be gathered continuously on the quality of FK4, and a decision will be facilitated at what time in the future the new system can be formed with profit. It is not unlikely that after the investigation of all observations made within the AGK3R program and after the completion of the SRS program, the new material might justify the revision of the present system and its extension to more stars and to a fainter magnitude limit.

\section{DISCUSSION}

G. van Herk: The Quito Observatory should be included in the list of those at which astrolabe observations useful for the correction of FK4 have been made. The desirability of observers publishing the apparent magnitude at which a star is actually observed (i.e. what screen, if any, is used) should be stressed in Section 4, 'Suggestions for Further Observations'. Otherwise the determination of errors dependent on magnitude by later investigators is made unnecessarily difficult. 